\title{
A Virtual Organisation for e-Learning
}

\author{
Nicola Capuano ${ }^{1,2}$, Pierre Carrolaggi $i^{3}$, Jerome Combaz Fabio Crestani ${ }^{4}$, \\ Matteo Gaeta ${ }^{1,2}$, Erich Herber ${ }^{5}$, Enver Sangineto ${ }^{1}$, Krassen Stefanov $^{6}$ Mikel \\ Vergara $^{7}$ \\ ${ }^{1}$ Centro di Ricerca in Matematica Pura ed Applicata,Università di Salerno, Italy \\ ${ }^{2}$ Dip. Ingegneria dell'Informazione e Matematica Applicata, Università di Salerno, Italy \\ ${ }^{3}$ Dept. Conseil-Recherche-Innovation, Greta du Velay, Le Puy-en-Velay, France \\ ${ }^{4}$ Dept. Computer and Information Sciences, University of Strathclyde, Glasgow, UK \\ ${ }^{5}$ CLICK\&LEARN Badegruber \& Partner GmbH, Linz, Austria \\ ${ }^{6}$ Dept. Information Technologies, Faculty of Math \& Informatics, University of Sofia, Bulgaria \\ ${ }^{7}$ European Software Institute, Bilbao, Spain
}

\begin{abstract}
We present in this paper the results of an European funded project Diogene, finished in October 2004, whose aim has been the design and the development of a distributed e-learning system able to perform several automatic actions such as course customization and information retrieval on the Semantic Web. The Diogene architecture is based on a network of specialized Organizations each of which has been realized as an independent Web Service.
\end{abstract}

\section{Introduction}

You cannot mandate productivity, you must provide the tools to let people become their best.

- Steve Jobs, CEO of Apple Computer

It is a well-known fact that many corporations would like to increase the global productivity of their production processes using e-Learning techniques as an alternative way to improve the effectiveness of their human resources. This general comment is also applicable to the SME's (Small-Medium-Enterprise) in the ITC (International Technology and Communication) market sector, in general lacking adequate personnel training.

Diogene is an European funded project under the 5th Framework Programme whose mission is to innovate the vocational and post-degree training in the ICT field making it more flexible and effective through the advanced use of the Information Technology. Particular interest is placed in the application of methodologies for distance learning and in the evaluation of their impact.

The activity of production, experimentation and research of Diogene focus in conjugating demands and didactic objectives of free-lance teachers, learning material providers and learners through well defined training strategies analysing, defining, planning and appraising the most suitable methodologies to support them.

Diogene objective is to design, implement and evaluate with real users an innovative training Web brokering environment for ICT individual training (but based upon a domain-independent platform) able to support learners during the whole cycle of the training, from the definition of Learning Objectives to the assessment of results through the construction of custom self-adaptive courses.

The system uses several state-of-the-art technologies such as: metadata and ontologies for knowledge manipulation, fuzzy learner modelling, intelligent course tailoring, co-operative and online training support. Besides, it includes a set of innovative features such as: dynamic learning strategies, Semantic Web openness, Web services for Learning Object handling and property rights management, Curriculum Vitae generation and searching facilities, free-lance teachers support, assisted Learning Objectives definition.

The system is accessible through the Web exploiting an ASP (Application Service Provisioning) methodology. Once a learner accesses the Diogene e-learning system, he/she can select a particular set of topics from an ontology of arguments and let the system to arrange a personalised self-adaptive course about chosen topics (personalisation is based on Learning Preferences). 
The Diogene e-learning platform includes eight courses covering different topics in three languages (English, Spanish and French). The following paragraphs describe briefly its innovative features with respect to commercial e-learning platforms presently available on the scene.

\section{Metadata and Ontologies for Knowledge Management}

All Diogene learning material is organised in Learning Objects indexed through IMS compliant metadata in order to let the system know what each one of them is about and how it can be used during the learning process.

To provide, also, information about Learning Objects relations and interdependency, Diogene applies Ontologies allowing to design abstract, simplified views of training domains. Within Diogene, ontologies are used to define and relate concepts of a training domain with three kinds of relations: (is_part_of, requires and suggested order) and, also, to link concepts to Learning Objects.

\section{Semantic Web Openness}

Another characteristic of Diogene e-learning system is its openness to the new Semantic Web paradigm. A key idea behind the project is, in fact, to not only rely upon ad-hoc generated learning material (i.e. high quality Learning Objects) but also to exploit the power given by the Web of the next generation to find useful and freeware learning material.

\section{Web Services for Learning Object Handling}

High quality learning material to be used in Diogene e-learning system is organised by content providers in several Learning Objects and maintained on their own Web servers. This means that such material is never imported on the main Diogene site avoiding, in this way, any property right related problem and giving content providers, in the same time, the possibility to manage contents (re-organise, update, delete) locally and not through complex HTTP or FTP based remote interfaces.

\section{Intelligent Course Tailoring}

A Diogene course is composed by an user selected set of learning goals (key concepts that the learner wants to learn) and by a learning path (a sequence of Ontology's concepts that has to be used to provide, to a specific learner, all necessary knowledge to fully understand chosen goals). Different learners can require different paths to learn the same goals depending on their Student Models. For this reason, Diogene provides an automatic learning path generation procedure: the learner can choose what to learn (goals) and let the system organise a personalised learning path for her/him. Such path can change dynamically during the learning process adapting to learner needs in relation to learner performed activities.

\section{Curriculum Vitae Search Engine}

Information about Diogene learner models is exportable in a standard format (PAI, LIP, etc.) in order to allow the possibility to create a standard Curriculum Vitae (CV) of the learner to be published, with respect to privacy requirements. Such curriculum includes information about: identification of biographic data, qualifications, learning related activities, competencies, language capabilities and preferences.

\section{Free-Lance Teachers Support}

Not only learning contents are indexed through Metadata but also other learning resources such as teachers are defined and described in that way. Free-lance teachers have, in fact, the possibility to subscribe Diogene and to describe (in a formal way) their professional abilities.

\section{The Diogene network}

Virtual Organizations (VO) are coordinated groups of individuals and institutions who collaborate towards a common interest and share, on the basis of some policies, a set of resources. Members of a VO can be geographically distributed and can access to the resources any time they are allowed. The element that characterizes the participants of a VO is the common purpose or business goal.

The Diogene project realised a Web Service based VO for e-learning. Diogene uses state-of-the-art technologies like metadata and ontologies for knowledge representation and management, fuzzy learner modelling, intelligent course tailoring and includes a set of innovative features such as 
dynamic learning strategies, Semantic Web openness, Web services for Learning Objects handling and IPR management, Curriculum Vitae maintenance and searching facilities, free-lance teachers support and assisted Learning Objectives definition.

The Diogene VO is populated by entities offering and consuming services. Such entities are grouped into two main categories: users (human beings that provide and consume services) and organizations (software components that provide and consume services).

\subsection{Diogene Users}

Diogene Users can be grouped into the following main categories.

- Learner: is a student registered in the Diogene VO. He/she acquires knowledge through learning experiences provided by Diogene specific learning services.

- Expert Learner: is a Learner skilled about some topics. He/she can offer mentoring support to other learners about such topics, usually for free.

- Freelance Tutor: is a professional tutor offering his/her specialized mentoring support to learners about specific topics usually under the payment of some price.

- Skill Searcher is an enterprise manager interested in hiring certified staff. He/she performs queries on skill repositories.

Further users like Content Providers, Knowledge Managers and Administrators are not discussed in this paper.

\subsection{Diogene Organisations}

Organizations of different type offer services with respect to the specifications of the Diogene VO and collaborate for the realisation of their own services. The kind of organisations supported by Diogene are summarised in the following list.

- Publishing Houses (PH). They store training content and provide remote access to it. They provide search and retrieval functions on the local repository via metadata-based queries.

- Web Catcher Agencies (WA). They are able to extract training content directly from Web and Semantic Web. Through a keyword-based text categorisation algorithm they can, where absent, semi-automatically extract metadata from textual learning objects ad link them to structures maintained by the Knowledge Agency.

- Tutor Agencies (TG). They work as entry point for freelance tutors, they manage the freelance tutor archive (containing tutor models) and provide searching facilities on such archive.

- Brokerage Offices (BO). They research, prepare and provide training offers for learners on demand, based on customisation information (learner model, learning strategies, price, learning goals). They collaborate with $\mathrm{PH}$ and WA in order to retrieve didactical material that best fit the customisation requests. They collaborate with TG to retrieve and provide freelance tutors that best fit learner needs.

- Training Agencies (TA). They provide the basic environment for learning experiences and tutoring activities. They are responsible for the delivery of courses and for the provision of course management and execution functions. They maintain learner models and are able to provide (on demand) course offers by exploiting services offered by BO.

- Knowledge Agencies (KA). They maintain and manage knowledge structures (concept dictionaries and ontologies) for the whole Diogene Network.

- Café (CA). They support social interactions, mentoring and information exchange by providing users a set of collaborative synchronous and asynchronous facilities. They automatically arrange groups among users of registered TA by individuating and grouping learners with similar needs and/or profiles.

- Skill Agencies (SA). They provide search engine capabilities on Learner Models Databases of registered TA in order to let third parties interested to hire certified staff to find qualified professional (with respect to privacy requirements). They maintain, moreover, requests statistics in order to rank the required competencies. 
- Bank (BA). It's a singleton organization that executes and logs transactions information occurred inside the Diogene VO. It interacts with an e-commerce engine to execute transactions involving real payment processes.

More instances of the same Organisations live inside the VO. The various instances stand on different hosts and are managed (administered) by different users. Figure 1 depicts some interactions between Diogene organisations.

The next chapters will focus on each of the Diogene Agencies.

\section{The Main Services}

In this section we present in more details some of the most important organizations explaining the way in which they work.

\subsection{Training Agency, Brokerage Office and Knowledge Agency}

These three agencies are the core of the whole net. A typical learning session works as follows.

A learner submits to the TA a query specifying the set of arguments she/he is interested to learn. The set of topics is chosen among the set of all the Domain Concepts of the Ontology, provided by the KA. Then, the TA sends to the KA the student's training query (called Target Concepts).

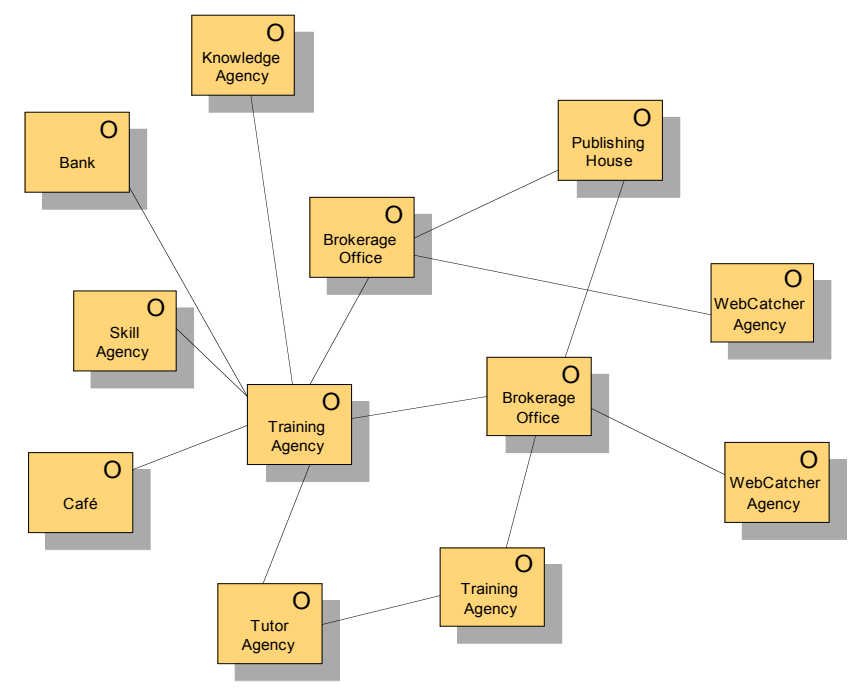

Figure 1. Interactions among different Diogene Organisations.

The KA, starting from the Target Concepts and using the pedagogical information on the domain of interest represented by its Ontology [Capuano et al. 02, Capuano et al. 03], generates a Learning Path, i.e. the set of all the Domain Concepts necessary to learn the input Target Concepts set. In the Learning Path there is no concept already known by the student (this can be verified using the Student Model), moreover the KA includes in the Learning Path all the necessary pre-requisite concepts and finally the Learning Path is ordered following the pedagogical instructions explicitly declared in the Ontology.

Once the Learning Path has been built, it is sent by the KA to the TA, which, in its turn, sends the Learning Path to the BOs. Each BO sends the Learning Path to all the PHs and WAs it is in contact with. Each $\mathrm{PH}$, received the Learning Path (i.e. an ordered set of Domain Concepts), sends back to the $\mathrm{BO}$ the set of Learning Objects Metadata related to at least one concept of the Learning Path. Analogously, each WA sends back to the BO all those documents (previously retrieved on the Web and off-line categorized) which are related to the concepts of the Learning Path.

Now each BO has a Learning Path (a list of necessary arguments), different sets of Learning Objects (coming from the contacted PHs and the WAs) and the Student Model (provided by the KA through the TA). Thus the BO is able to select the best training offer for the student. This is done by comparing the available Learning Objects with both the Student Model (in order to select the ones nearest to the student's learning preferences) and the student's price request. The output of each $\mathrm{BO}$ is a Presentation, which is an ordered set of Learning Objects explaining the whole Learning

$1^{\text {st }}$ International Kaleidoscope Learning GRID

on Distributed e-Learning Environments Workshop 
Path. The Presentation is finally sent to the TA which presents it to the student. The student will select the best Presentation among all the Presentations provided by the different BOs contacted by her/his TA.

\subsection{Web Catcher Agency}

The purpose of the WebCatcher Agency (WA) is to discover free content on the Web, and to provide this to learners through the Diogene system. This free content may be of lower quality than the training materials provided by registered content providers, but can be used as additional material to aid learners.

In order to discover this material, the WA performs searches on the Web and Semantic Web using a mixed approach based on keywords and ontologies, whose details are reported in [Villa et al. 04a]. This approach enables to bypass compatibility problems between different ontological representations of the same domain that can be found on the Semantic Web and, at the same time, offering a search service, matching learning objects that can be found on the Web to users' requests.

The WA also uses a keyword-based text categorisation algorithm to enable, where absent, to automatically extract metadata from textual learning objects and to link them to Diogene's ontology concepts [Villa et al. 04b].

The WA is directly linked to Diogene's Brokerage Office. The Brokerage Office collaborates with the Publishing House and the WA in order to retrieve didactical material that best fits the customisation request.

\subsection{Publishing Houses}

The Publishing house is a Diogene Organization focused on cataloguing and managing high quality didactical material. There could be more than one Publishing House inside DIOGENE Network. The didactical material is composed by Learning Objects (LO), specified in term of content (i.e. HTML, PDF) and metadata (IMS-LOM). For each learning object the Publishing house stores content in LO Content Repository, stores metadata in LO Metadata Repository and updates indexes of LO Metadata. Other Diogene organizations can demand learning objects from Publishing house and can get them in the form of a content package. For that purpose they have to use the services of the Brokerage office.

Publishing house provides the following web services:

- Store package of learning objects and update the metadata indexes - unpacks learning objects pack into several learning objects composed by content and metadata. For each learning object stores content in LO Content Repository, stores metadata in LO Metadata Repository and updates indexes into LO Metadata Repository.

- Retrieve personalized learning object's list - searches the LO Metadata that best matches the input LO Metadata Query. All LO Metadata founded will be returned in a LO Metadata List. The LO Metadata Query is represent as XML structure. Every Query is composed by one or more query nodes. The node's value is matched against every LO Metadata. If match succeed then the Metadata is selected. The process returns a union of all selected Metadata.

Deliver learning objects - This process creates the Learning Object Pack, that contains the Content and Metadata of the LO required, and returns it.

\subsection{Tutor Agency}

The Tutor Agency is a Diogene Organization that enables the storage and retrieval of information about tutors, their models, and their schedules.

Companies registered into the Diogene Network can perform queries on a Tutor Agency in order to retrieve tutors with specific skills such as language, education level and areas of experience.

Tutor Agency has several important features:

- Support the management of tutor profiles (each tutor can add, view, update and delete private information such as contact information).

- Support the management of tutor schedules (each tutor can add, view, and update his/her schedule).

- Support the management of tutor model - that is the language, level of education, and skills in specific subjects.

$1^{\text {st }}$ International Kaleidoscope Learning GRID

on Distributed e-Learning Environments Workshop 
- Support the tutor searching activity that allows students to searcher and find a tutor that best fits the criteria such as language, level of education and skills in a specific area of interest.

In order to use the Tutor Agency, the user have to be registered inside the Skill Agency from the Diogene Network Reception.

\subsection{Skill Agency}

The Skill Agency can be queried by third parties interested to hire specialized staff. The Skill Agency (SA) receives from the Training Agency (TA) a data base of Student Models. In this data base the SA will search for people to propose as answer to the query. Searching in the data base works as follows. The input query specifies a set of Domain Concepts (see Section 3.2), i.e. a set of topics/skills in which the external company is interested as the wished application curriculum. Since the Student Model of each learner contains, for each Domain Concept, the representation of the knowledge degree shown by the learner on that subject, the SA can rank the Students' Models provided by the TA by selecting in each Student Model the Domain Concepts specified in the query and then maximizing the sum of all the knowledge degree corresponding to each student. Finally, the SA will propose to the skill searcher the ranked list of learners with the possibility to contact them.

\section{Examples of complete courses provided by Diogene}

Eight courses have been adapted from existing linear courses. Courses have been split in many LOs to make it Diogene compliant. It has been necessary to rewrite some parts of the content, to ensure that each LO is atomic (no reference is made to another LO or to another concept). A Main Ontology covering the ICT domain was created to index these LOs, based on the ACM-CCS classification (Computing Classification System). ACM was chosen for different reasons : it has a widespread ICT taxonomy, it is easily expressed using the Diogene relations, and it is directly linked with the ACM \& IEEE Computing curricula guidelines, describing how courses in the field of computing have to be arranged in the suitable training programmes at various levels. The actual Ontology contains and extends the CCS, using the Requires/Suggested Order/Has part relations to organise 1652 concepts.

The eight genuine courses cover a wide range of ICT concepts among the Ontology : how to secure data access, processing and transmission for SMEs dealing with information systems; the theory of digital images and how ICT workers can exploit them; how to set up a basic development environment to develop dynamic web pages with the PHP language; Business Process Management is a set of methods to help better decision making; some other courses cover software engineering, like 'COTS' approach for large-scale software development, Balanced IT Scorecard for software process improvement, object oriented analysis and design which covers all the UML notations, and a set of LOs about the fundamentals aspects of XML.

The content producers used the KMS tool to make interactive exercises used by the system to test the learners on each LO. Test and LO have been meta-tagged with the same tool regarding their content: language, interactivity level, typical learning time, learning styles, etc. All LOs and test are available in English and optionally in the content producer's languages (Spanish and French for the experimentation). Diogene deals the content in the language of the learner regarding its profile whether localized LOs are available. It is easily expendable to other languages. Actually the experimentation includes a total of 716 LOs.

\section{Evaluation results}

Experimentation and evaluation of the DIOGENE system was conducted between April and October 2004 and built up on several use scenarios that realised a real life learning environment in a VO. The results of this process proved that DIOGENE has had a significant impact on the brokerage of ICT courses and the support of the learning life-cycle by use of the underlying organisations.

One of the major benefits that DIOGENE enables, as many evaluators pointed out, is providing the available courses on a resource list directly to concepts of a structured knowledge domain, and supporting fundamental processes of the whole learning life-cycle. Learners proved highly satisfied with the allocation of courses they achieved by use of their profile definitions $(72 \%)$ and the adequacy of the courses that were suggested by the system to represent their lack of knowledge (78\%). Tutors evaluated DIOGENE as a useful platform for the support of their tutorial services. It

$1^{\text {st }}$ International Kaleidoscope Learning GRID

on Distributed e-Learning Environments Workshop 
was appreciated from tutors that DIOGENE allows a mechanism that supports collaboration with learners and that enables tutors to support the learning process. Skill searchers confirmed the value of the services related to skills requests in the process of higher qualification and/or recruitment.

According to the innovative aspects that can be associated with DIOGENE, evaluators assessed DIOGENE to be a highly innovative (63\% of evaluators) and highly useful (94\% of evaluators) system that provides easy to use and good quality ( $91 \%$ of evaluators) services of which they would want to make use again once DIOGENE will be available as a commercial service $(87 \%$ of evaluators). It was also clearly found out that where constant communication and interaction between the stakeholders of the VO was held, learning outcomes and progress could be improved and processes made easier as well as more transparent to stakeholders. Figure 2 shows some evaluation criteria on which DIOGENE had been evaluated.

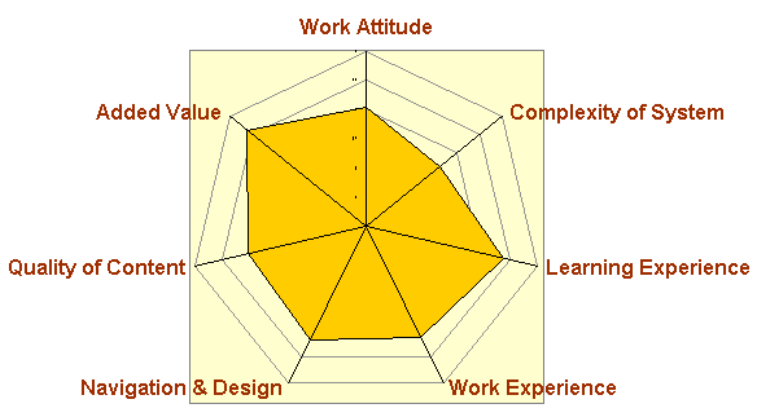

Figure 2. The added value of DIOGENE

The evaluation results demonstrate that DIOGENE as a VO has a good level of utility for providing learning resources of various aspects and domains and supporting the learning life-cycle in a distributed environment, but complexity of knowledge domains is an issue that requires good methods and concepts in order to be translated to users. The major impact of the work of the DIOGENE project and evaluation outcomes will be on taking forward the results and lessons learnt from the project and improving further such Virtual Organisations to support the processes of learning.

Applied Methodology: Evaluation involved 170 users of various roles and types (unskilled / skilled / expert users with diverse language backgrounds) from 6 European countries and was based on a range of well-known evaluation methods: use scenarios, performance recordings, online questionnaires, interviews, and document analysis.

\section{Conclusions}

We have presented a distributed e-learning system created during the European funded project Diogene (5th Framework Programme, Information Society Technologies, contact number IST-200133358, see [Diogene] for more details). Diogene is based on a network of independent organizations each of which realized as a Web Service. These agencies provide several e-learning and related facilities, such as course delivering, course customization, free document retrieval and categotization on the Web and on the Semantic Web, learning objects' repositories based on a metadata indexing, human tutor support and external skill search. During the project we also have built a complete Ontology of the ICT topics, [Stefanov \& Todorova 03], used by the system as domain knowledge representation, which completes the ACM taxonomy of the Computer Science. Moreover Eight courses have been adapted from existing "traditional" courses splitting them in different Learning Objects and indexed using Semantic Web standards. Finally, the project has been evaluated using 170 users of various roles (learners, tutors, ...) with very encouraging results.

\section{References}

[Capuano et al. 02] Capuano N., Gaeta M., Micarelli A. and Sangineto E. An integrated Architecture for Automatic Course Generation. Proceedings of the IEEE International Conference on Advanced Learning Technologies (ICALT 02), Kazan, Russia, 2002.

[Capuano et al. 03] Capuano N., Gaeta M., Micarelli A. and Sangineto E., An Intelligent Web Teacher System for Learning Personalization and Semantic Web Compatibility, Proceedings of the Eleventh International PEG Conference, St. Petersburg, Russia, 28 June-1 July 2003.

$1^{\text {st }}$ International Kaleidoscope Learning GRID

on Distributed e-Learning Environments Workshop 
[Diogene] Diogene: a Training Web Broker for ICT Professionals (IST-2001-33358):

http://www.diogene.org.

[Stefanov \& Todorova 03] K. Stefanov, K. Todorova. Computing Ontology Creation. In Proceedings of International Congress MASSEE2003, pages 40-49, Borovets, Bulgaria, 15-21 September 2003.

[Villa et al. 04a] R. Villa, R. Wilson, and F. Crestani. Ontology Mapping by Concept Similarity. In Proceedings of ICDL 2004, International Conference on Digital Libraries, pages 666-674, New Delhi, India, February 2004.

[Villa et al. 04b] R. Villa, R. Wilson, and F. Crestani. Automatically Attaching Web Pages to an Ontology. Proceedings of IPMU 2004, International Conference on Information Processing and Management of Uncertainty in Knowledge-Based Systems, pages 528-535, Perugia, Italy, July 2004. 